\title{
LA RESILIENCIA EN FAMILIAS QUE VIVEN LA DISCAPACIDAD, DESDE UN ENFOQUE CENTRADO EN LA FAMILIA
}

\author{
RESILIENCE IN FAMILIES THAT \\ EXPERIENCE DISABILITY, FROM A \\ FAMILY-CENTERED APPROACH
}

Emma Verónica Santana Valencia

Doctora en Ciencias para la Familia por ENLACE México. Directora Académica del Posgrado en Orientación y Desarrollo Familiar de la Universidad Popular Autónoma del Estado de Puebla y candidata al Sistema Nacional de Investigadores (SNI). emmaveronica.santana@upaep.

\section{RESUMEN}

Este trabajo es el resultado de una investigación doctoral, cuyo propósito es conocer cómo se construye la resiliencia en las familias que viven la discapacidad en un hijo. Los cuestionamientos son ¿cómo se construye la resiliencia familiar frente a la discapacidad de un hijo?, ¿cuál es la dinámica del sistema familiar frente a la discapacidad?, ¿qué elementos de la resiliencia se observan en las familias que experimentan la discapacidad en un hijo? El supuesto es que toda familia que tiene un hijo o hermano con alguna discapacidad es capaz de ser resiliente, de 
manera que es apta para afrontar la adversidad, identificar sus fortalezas y empoderarse en función de sus elecciones. Se emplea una metodología cualitativa, con un diseño de estudio de caso, a través de una entrevista a cinco familias. Se concluye que la resiliencia es un proceso dinámico que se manifiesta en las familias durante la vida en grupo.

Palabras clave: familia, discapacidad, resiliencia y enfoque centrado en la familia.

\section{ABSTRACT}

This work is the result of a doctoral research, which purpose is to know how resilience is built in families that live with a child with disabilities. The questions are: How is family resilience built around a child's disability? What is the dynamic of the family's system facing disability? What elements of resilience are observed in families that experience disability with a son of their own? The premise of the study is that every family that has a child or sibling with a disability is capable of being resilient, so that the family can face adversity, identify their strengths and empower themselves based on their choices. To assess this claim, a qualitative methodology was applied with a study-case design and in depth interview with five families. Findings support the conclusion that resilience is a dynamic process that manifests itself in families during life.

Key words: family, disability, resilience and family-centered approach.

\section{INTRODUCCIÓN}

Todas las personas son parte de una familia, independientemente del lugar donde habiten, la cultura que posean, la comunidad donde se desenvuelvan y la estructura que posean; esto hace que los humanos cuenten con cierto conocimiento desde la propia experiencia, debido a que como persona se ha nacido y crecido dentro del colectivo básico de 
toda sociedad, llamado familia. En ella se ofrece seguridad, se otorga aceptación incondicional, haciendo de ello la manifestación más clara de su función como formadora de personas, es decir, la humanización. De manera especial cuando la discapacidad se manifiesta en un hijo, la familia se ve golpeada por una serie de eventos, sentimientos y emociones diversas, los cuales deberán lograr su cauce.

Para alcanzar un equilibrio al interior de la familia se requiere de un trabajo profundo de aceptación y afrontamiento de la nueva realidad, que ha comenzado a mirarse desde un lugar de oportunidad. Es factible reconocer que no todo es malo ante este hecho. Pero para desarrollar este proceso, es necesario ser resiliente y ¿qué es esto?, los expertos (Kotliarenco et al., 1997, Villalba, 2003, Grotberg, 2006, Palomar y Gaxiola, 2012) la denominan como la capacidad de hacerle frente a la adversidad y salir fortalecido de ella. De modo que en el caso de estas familias es posible observar que, con el pasar del tiempo y el desarrollo de capacidades, logran avances agigantados en su propio crecimiento.

Por tal motivo la presente investigación tiene como propósito conocer la manera en que se construye la resiliencia en las familias que viven la discapacidad en un hijo, mediante una entrevista a los integrantes de las mismas. De los resultados obtenidos podrá describirse la dinámica que poseen, así como los elementos claves de la resiliencia. Todo desde una mirada centrada en la familia, ya que ella es el foco principal de atención, por su influencia en cada uno de sus miembros.

En un niño diagnosticado, los padres son el referente principal para su apoyo natural, debido a que pasan más tiempo en el hogar, a causa de los obstáculos que se les imponen. Las familias que experimentan este hecho, desarrollan una fuerte tensión entre el ser y el deber ser, cuestionando su quehacer. Paralelamente, la familia empleará su bagaje relacionado a la discapacidad e irá presentando una serie de emociones distintas. Lo anterior revela una potencial familiar para enfrentar las complejidades de la vida, donde pueden resurgir con una existencia inédita y fortalecida, autodeterminada a partir de esa experiencia, para demostrarse que es capaces de enfrentar la situación, generando resiliencia desde su interior (Peralta y Arellano, 2010). 
Por lo tanto, se parte del supuesto que toda familia con un hijo o hermano con alguna discapacidad es capaz de ser resiliente, de manera que es apta para afrontar la adversidad como grupo, identificar sus fortalezas y empoderarse en función de sus elecciones. Así, se indaga en un fenómeno social incorporado a la realidad humana, desde una perspectiva construida a partir de un enfoque centrado en la familia (ECF). Esto implica considerarla capaz de enfrentar esta circunstancia de vida, pero desde un lugar donde la premisa de la resiliencia permite suponer que se priorizan los objetivos positivos, se promociona el desarrollo sano, se generan competencias y se previenen problemas futuros (Grau, 2013).

\section{LA FAMILIA, ANCLAJE DE LA RESILIENCIA}

La familia, sigue siendo el pilar más firme que sustenta a toda comunidad, particularmente en la colectividad humana. Para su comprensión desde el enfoque sistémico (Minuchin, 2008; Eguiluz, 2003; López y Escudero, 2003; y Macías, 2012), se determina la importancia de conceptualizar y estudiarla con una visión completa, donde se le comprende como sistema. León (2015) afirma que esta perspectiva consiste en observar a la persona como una pieza del sistema familiar, analizándola como una organización abierta con estructura, funciones y a su vez subsistemas. A partir de esta visión integral de la familia, debe comprenderse que cuando la discapacidad se presenta dentro del sistema, la dinámica interior y exterior, comienzan a mostrar comportamientos peculiares. Garzón (2010) comenta que la condición de discapacidad suscita una serie de hechos, mandatos, prácticas y creencias provenientes de la sociedad para con las personas, que suelen ser injustas e incorrectas, provocando en la familia una serie de reacciones en diversas direcciones, dependiendo de su propia historia y sus rasgos particulares: rebeldía, conformidad o adaptación.

En la actualidad se pone de manifiesto que también son capaces de observar oportunidades, es decir, descubren que este hecho les ofrece circunstancias de vida particulares y suelen afrontar la discapacidad desde un lugar distinto, pues generan significados, valores y formas de ver la vida con un sentido único (Aznar y González, 2008; Peralta y Arellano, 
2010, 2012). Esto implica que no niegan la existencia de las múltiples y diversas demandas que genera la presencia de la discapacidad, pero son capaces de enfrentar, así como de reconocer los aspectos positivos en sus vidas (Peralta y Arellano, 2010). Esto queda demostrado en las diversas investigaciones sobre la visión positiva con la coexistencia de aspectos negativos, vislumbrando una nueva realidad familiar, pues existe un ajuste entre todos los miembros del grupo (Villalba, 2003 Ortega et al., 2007; Bayat, 2010; Suriá, 2013). Este ejercicio familiar evidencia la resiliencia como un hecho tangible.

Debido a la complejidad que encierra en sí misma, la resiliencia cuenta con un sinfín de definiciones, pues en su evolución ha sido considerada como capacidad, proceso y paradigma (Luthar y Zigler, 1991; Werner y Smith, 1992; Rutter, 1993; Osborn, 1993; Grotberg, 1995; Puig y Rubio, 2011); por lo tanto, es una capacidad que, a través de un proceso, permite orientar una adversidad de manera funcional.

Ahora bien, la presencia de la resiliencia en estas familias puede comprenderse desde la influencia que se posee al contar con un ambiente favorecedor generado por la misma, debido a que algunos estudios demuestran que el foco de atención es reconocer y comprobar los aportes positivos de la familia a la resiliencia, con el pasar del tiempo (Patterson, 1991; Werner y Smith, 1992; Rutter, 1985; Anthony, 1987; Garmezy, 1991). Y de manera más actual, se perfila una línea de investigación sobre la resiliencia familiar ante las adversidades (McCubbin et al., 2002; Walsh, 2004; Grotberg, 2006; Delage, 2010).

\section{RESILIENCIA FAMILIAR Y EL ENFOQUE CENTRADO EN LA FAMILIA (ECF)}

Estudios sobre la relación, los vínculos y las manifestaciones que existen entre la familia y la discapacidad de un hijo (Araya, 2012; Mercado et al., 2012; Mora, 2012; Vargas, 2013; Roque y Acle, 2013); explican la complejidad en las demandas de ser padres, pero también muestran aspectos positivos, generando un sentido de vida y fortalezas para enfrentar esta situación. 
De modo paralelo es posible observar en los padres la generación de estrategias sólidas y múltiples con altos niveles de creatividad (De Andrade y Da Cruz, 2011), así como reportan aspectos positivos en la crianza de un hijo con discapacidad (Blacher y Baker, 2007; Trute, et al., 2007), éstos propician una vida enriquecida de experiencias significativas, presencia de valores profundos, con una espiritualidad particular y completa.

La resiliencia familiar se genera en su interior, por ello hay que considerar reposicionar a la familia en el centro, como la primordial base de información y saber del hijo con discapacidad (Dempsey y Keen, 2008). Se requiere un enfoque particular de intervención, donde el objeto de primer orden sea capacitar a las familias, con lo cual se promueva un apoyo y fortalecimiento en su interior. Dunst, Trivette y Deal (1989, 1994 y 1997) denominan a este enfoque Family Centred Approach o bien Family Centred Practices; el cual se focaliza en las familias como agentes activos en el desarrollo de sus hijos. También es considerado como un modelo que cuenta con la visión de la familia para el futuro, incluyendo a sus hijos con discapacidad de modo total.

Autores como Giné, Gracia, Vilaseca y Balcells (2009) denominan a este tipo de trabajo «enfoque centrado en la familia», un enfoque de intervención construido en función de un conjunto de creencias, valores y acciones, respetando y ejercitando a la familia en la toma de decisiones (Dunst, Trivette y Hamby, 2007).

\section{METODOLOGÍA}

La investigación se realizó en la capital del estado de Puebla en México. Los hijos de las familias participantes asistían o habían asistido de manera regular a alguno de los dos centros de apoyo denominados AMAD (Asesoría para el Manejo Adecuado de la Discapacidad) o CEADI (Centro de Evaluación y Atención para el Desarrollo Integral del Individuo); los hijos contaban con un diagnóstico de discapacidad desde su nacimiento y se encontraban en la niñez intermedia. 
Se contó con la colaboración de cinco familias y sus integrantes, involucrados con el hijo, hermano o sobrino con discapacidad. A continuación puede observarse la descripción de las familias participantes.

\section{Tabla 1. Descripción de las familias.}

\begin{tabular}{|c|c|c|c|c|c|}
\hline Familia & $\mathrm{F} 1(\mathrm{IC})$ & $\mathrm{F} 2(\mathrm{Dm})$ & $\mathrm{F} 3(\mathrm{Jr})$ & F4(Ia) & $\mathrm{F} 5(\mathrm{Gu})$ \\
\hline $\begin{array}{c}\text { Estatus } \\
\text { socioeconómico }\end{array}$ & Alto & Bajo & Medio & Medio alto & Medio \\
\hline Nivel educativo & Licenciatura & Básico & Técnico & Licenciatura & Licenciatura \\
\hline Tipo de familia & Reconstituida & Monoparental & Monoparental & Monoparental & Monoparental \\
\hline $\begin{array}{l}\text { Estado civil de } \\
\text { los padres }\end{array}$ & Casados & Separados & Separados & Divorciados & Viuda \\
\hline Participantes & 4 & 2 & 2 & 2 & 2 \\
\hline $\begin{array}{l}\text { Diagnóstico de } \\
\text { la discapacidad }\end{array}$ & $\begin{array}{l}\text { Mielomeningocele } \\
\text { con hidrocefalia } \\
\text { compensada }\end{array}$ & $\begin{array}{c}\text { Daño } \\
\text { neurológico } \\
\text { con } \\
\text { discapacidad } \\
\text { motora }\end{array}$ & $\begin{array}{l}\text { Agenesia del } \\
\text { cuerpo calloso } \\
\text { con quiste de } \\
\text { lado izquierdo e } \\
\text { hipoacusia }\end{array}$ & $\begin{array}{l}\text { Trastorno } \\
\text { general del } \\
\text { desarrollo no } \\
\text { específico }\end{array}$ & $\begin{array}{l}\text { Ceguera/ } \\
\text { Débil } \\
\text { visual con } \\
\text { discapacidad } \\
\text { motora }\end{array}$ \\
\hline
\end{tabular}

Fuente: Elaboración propia.

Se desarrolló una metodología cualitativa, mediante un diseño de estudio de caso en dos fases, a través de las cuales se dio seguimiento al conocimiento e integración de la estructura y su contenido. En la primera fase se establecieron los mecanismos teóricos que fortalecieron la construcción de la estructura general de la investigación, con datos pertinentes de los modelos de resiliencia, seleccionando tres que poseen una interrelación representativa con el presente fenómeno de estudio: modelo personal, familiar y comunitario. Luego, se eligieron las dimensiones por conocer; a partir de estos argumentos fue posible desarrollar un conjunto de unidades de contenido, vinculadas al conocimiento de la construcción de la resiliencia. 
Se realizó un acercamiento inicial con los informantes a través del instrumento «Escala de resiliencia mexicana» (RESI-M) (Palomar J., y Gómez N., 2010), como apoyo para la construcción conceptual de categorías y subcategorías.

La segunda fase de la investigación desarrolló la técnica de entrevista a profundidad a través de una guía, diseñada en función de la información obtenida hasta este momento. Ésta fue semiestructurada y contó con un formato de diecisiete interrogantes, generados en función de los diversos elementos que los expertos consideran propios de la resiliencia. Esta guía fue evaluada por parte de cuatro investigadores del área.

Con la guía establecida (acorde a las observaciones sugeridas), se procedió a organizar las preguntas en categorías y subcategorías establecidas previamente, con el propósito de contar con una organización clara de la información que se quería rescatar de las voces de la población participante. Se determinaron los tres campos generales de Walsh (2004), surgiendo seis categorías y ocho subcategorías para el análisis.

Después se desprendió la posibilidad de asignar preguntas del formato de entrevista, las cuales generaron conocimiento profundo de la realidad de estudio y la construcción de la resiliencia en las familias con un hijo con discapacidad, siendo parte del tratamiento designado por la presente investigación. A continuación se muestra de forma esquemática este proceso de construcción. 


\section{Figura 1. Proceso de investigación.}

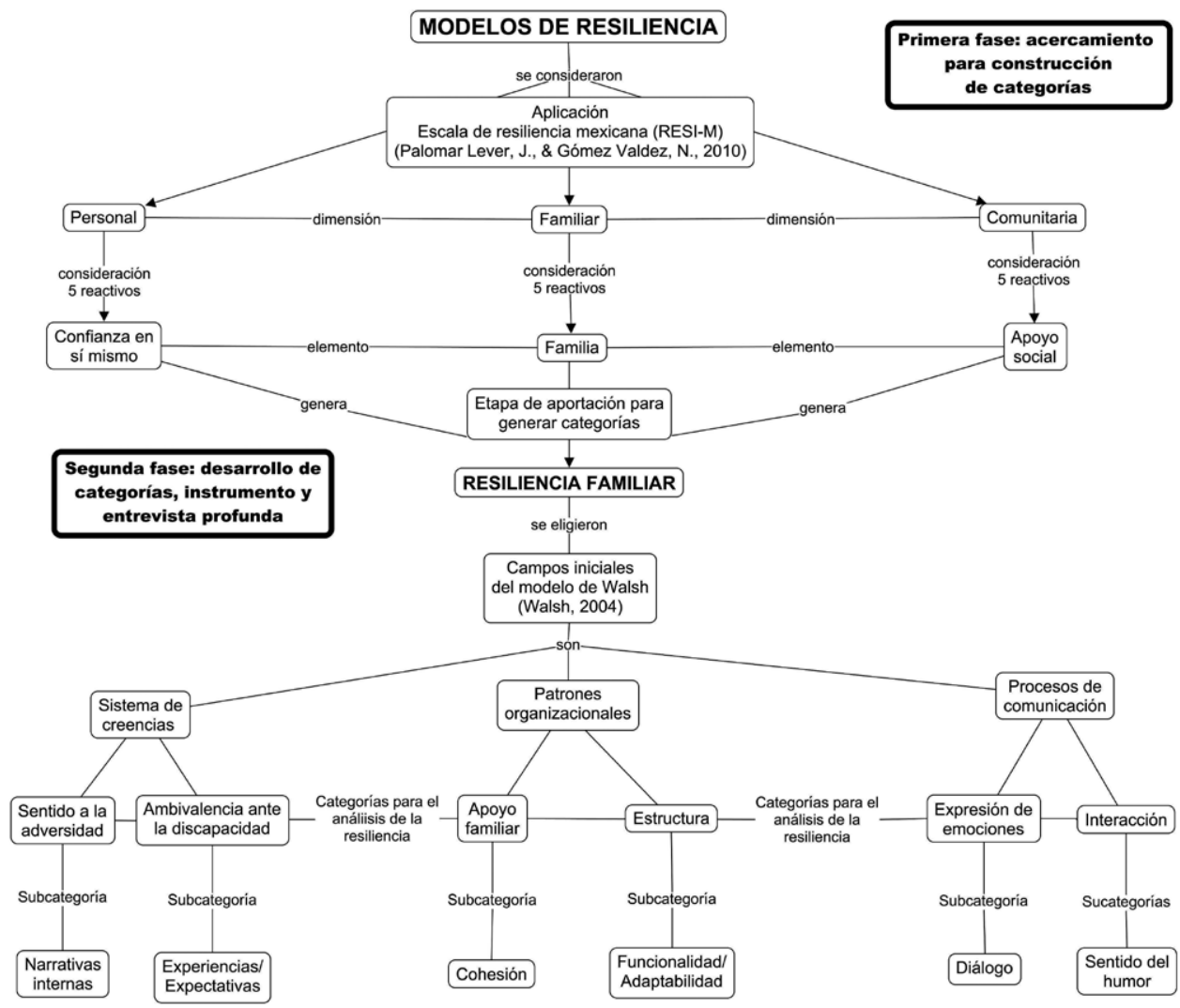

Fuente: Elaboración propia.

Las entrevistas a profundidad se llevaron a cabo en un período de siete meses. Tuvieron un margen de duración aproximada de treinta minutos (30 minutos) a dos horas con treinta minutos (2:30 horas), según la persona que participaba en cada momento. La entrevista semiestructurada con 17 ítems se aplicó en 5 familias, contando con un total de 12 personas entrevistadas. 
A partir de esta aplicación se obtuvieron un total 204 respuestas por pregunta. Éstas se analizaron a detalle, revisando cada una y eligiendo 13 preguntas claves, las cuales aportaban a las unidades de significado. Con ello se obtuvieron 156 respuestas, las cuales ofrecieron información pertinente.

La captura de la información obtenida se integró a través de una codificación abierta a partir de los testimonios de los participantes. Las respuestas contaban con datos vinculados a la información buscada; éstos se analizaron a detalle generando una selección de los datos más representativos. Este proceso se llevó a cabo en el total de los participantes, contando al final con un análisis de las 156 respuestas previamente mencionadas.

Posteriormente se estableció una codificación axial que incluía las categorías y subcategorías, relacionando entre sí la información para lograr descubrir la explicación, el sentido y significado de la realidad estudiada, a través de un proceso dinámico y flexible donde se profundizó en la información compartida, con un análisis de las palabras claves antes obtenidas.

Esta acción permitió reconocer a detalle las seis categorías previstas (sentido proporcionado a la adversidad, ambivalencia ante la discapacidad, apoyo familiar, estructura, expresión de emociones e interacción), así como las ocho subcategorías (narrativas internas, experiencias/expectativas, cohesión, funcionalidad/adaptabilidad, diálogo y sentido del humor), además de las categorías emergentes las cuales fueron cuatro (singularidad, valores, red de apoyo y espiritualidad).

\section{RESULTADOS Y DISCUSIÓN}

A partir de los testimonios de los participantes, se obtuvieron una serie de evidencias acerca de la construcción de la resiliencia en familias. Con respecto a la presencia de la discapacidad, destaca que aunque todos los diagnósticos de los hijos o hermanos fueron diversos en la presente investigación, ello no generó diferencias extremas en la formación de la resiliencia, pues los participantes reportaron sentirse afectados 
en un primer momento por el diagnóstico recibido, pero con el tiempo cada miembro robusteció sus vínculos familiares, desencadenaron recursos para enfrentar la nueva experiencia de vida, fortalecidos por su familia como el anclaje que les da fuerza ante esta circunstancia.

Uno de los primeros elementos reconocidos en esta fase inicial de la presencia de diferentes discapacidades, radica en la aceptación la cual, desde la experiencia de los miembros de la familia, se convierte en el medio más fuerte para enfrentar esta situación. Por lo tanto, las familias no niegan sus dificultades ante la discapacidad de un hijo o hermano, pero de forma paralela se mantienen optimistas y unidos, generando un frente común ante el hecho que les tocó vivir.

La experiencia anterior, recuperada a través de las familias, presentó una relación con el ECF, el cual sostiene que — sin negar la presencia de diversas exigencias de carácter cognitivo, socioeconómico, físico y emocional- la discapacidad desencadena aspectos favorables en el grupo. A continuación se describen los hallazgos obtenidos según las categorías y subcategorías establecidas, además de las categorías emergentes.

\section{SISTEMAS DE CREENCIAS}

En la entrevista, las familias reportaron que dentro del sistema de creencias, constituido por el sentido a la adversidad, las narrativas internas, la ambivalencia ante la discapacidad y las experiencias paralelamente con las expectativas, fueron elementos que inicialmente ejercieron un impacto determinante para generar resiliencia, ya que se manifestaron durante el primer enfrentamiento con la discapacidad y, a partir de ahí, generaron un conjunto de ideas, concepciones y representación de la vivencia.

Refieren que han encontrado un sentido a su vida, reconocido las nuevas posibilidades como una forma de lograr metas, y asumen esta experiencia como un reto del cual han aprendido y deberán seguir aprendiendo. Así, la vivencia de la discapacidad en un ser querido, les ha otorgado enseñanzas insustituibles, impregnando de significados a sus propias vidas, tanto a nivel personal como familiar. 
El conjunto de las narrativas internas obtenidas de los participantes, distinguieron una serie de verbalizaciones de carácter positivo, las cuales aportan a la construcción de la resiliencia. Al analizar la ambivalencia ante la discapacidad de las diversas personas entrevistadas, se encontró que la mayoría vive en un permanente tránsito entre la presencia de sentimientos y emociones, las cuales se ubican dentro de un espectro de sensaciones negativas a positivas, y viceversa. Este proceso permite a los involucrados construir y deconstruir su sentir.

De manera conjunta a lo descrito anteriormente, es evidente una relación entre la ambivalencia reportada por las familias, así como los testimonios que se relacionan con las experiencias vividas y las expectativas deseadas. También de modo general se pudo distinguir que sus experiencias se fincan en un carácter de lucha, reconocimiento y aprendizaje, gestando una serie de expectativas positivas, ambiciosas y optimistas, sobre todo centradas en lo que son capaces de lograr, particularmente como grupo.

Por lo tanto, las familias que han hecho uso de sus experiencias, manejan mejor la discapacidad, desencadenando con ello un conjunto de expectativas ancladas en la realidad vivida. Ello suscita una esperanza realista, debido a que la esperanza tiene una necesidad de veracidad, así como el realismo requiere de la esperanza, de modo que se articulan dos realidades como un reto, posibilitando que las familias decidan continuar (Vanistendeal, 2014). Este proceso construye la resiliencia familiar.

\section{PATRONES ORGANIZACIONALES}

Los patrones organizacionales incluyen: apoyo familiar, cohesión, estructura y funcionalidad, paralelamente con la adaptabilidad; los testimonios de las familias participantes, describieron estos patrones organizacionales desde el papel que les tocaba representar en sus propios contextos, en el campo de la organización. En el interior de las familias que viven la discapacidad en un hijo, hermano o sobrino, se preparan y colaboran de diferente manera, debido a la necesidad de hacer frente a la realidad que experimentan. A partir de esta experiencia, se puede detonar en los miembros de la familia cierto desajuste, de manera que 
la familia debe procurar organizarse desde el primer momento $y$, con el pasar del tiempo, desarrollar un entorno de seguridad (Calero, 2012).

En todas las familias que se entrevistaron, existió la presencia de una sólida figura materna, la cual se manifestó, sin conocer el término, como autodeterminada, organizada, acorde a su propia realidad, conocedora en la medida de lo posible de la discapacidad de su hijo o hija, ejerciendo un liderazgo dentro de su sistema familiar, así como la responsabilidad de distribuir funciones entre los otros miembros del grupo, con un conocimiento profundo de cada uno, y dispuesta además ella misma a seguir en el aprendizaje.

La categoría de apoyo familiar, aportó a la construcción de la resiliencia en la manera en que la familia se hacía sólida y se fortalecía, de acuerdo con la identidad de cada integrante y la singularidad como grupo, trabajando todos por un mismo objetivo: crecer como familia para la mejora e inclusión del familiar con discapacidad.

Los testimonios recogidos convergen en una realidad común, suscitada por la presencia de la discapacidad, de modo que, al ser parte de su vivencia, se convierte en un motivo de unión que desarrolla cohesión en la familia. Esto genera en los miembros un fortalecimiento de las relaciones según su papel, padre-hijo, madre-hijo, hermanahermano, esposo-esposa, madre-hija, padre-hija, por mencionar algunas. Esto evidencia lo que expertos en el estudio de la familia sostienen al afirmar que es un sistema vivo, único y con capacidad de autorregulación (Eguiluz, 2003, López y Escudero, 2003).

Al analizar la categoría de estructura se observó que la mayoría eran familias monoparentales, donde la autoridad recaía en su totalidad en la figura de las madres. Ellas han aprendido a enfrentar la discapacidad de sus hijos de modos diversos, debido a las experiencias adquiridas a través de sus propias historias de vida. Construyen su soporte a través de los otros hijos, convirtiéndose ellos en su sostén más valorado.

Las subcategorías de funcionalidad y adaptabilidad, surgen de la estructura y aportan a la organización del sistema familiar; estos dos aspectos se encuentran vinculados, debido a la cercanía que poseen 
para enfrentar la discapacidad de un hijo o hermano, durante la cotidianeidad.

La mayoría de las familias que participaron en este estudio, afirmaron que para hacer la vida más funcional requirieron en un principio de la integración y colaboración de todos los miembros del grupo, lo cual aunado a la categoría anterior, propicia observar la importancia de la familia como el mayor cimiento psicoafectivo y físico, para el quehacer diario, cuando de discapacidad se habla.

La flexibilidad es un elemento que aporta a la subcategoría de adaptabilidad, debido a que es una habilidad que contrarresta las diversas situaciones que pueden manifestarse en el acontecer de estas familias. Sobre la funcionalidad familiar en la discapacidad, se observa un funcionamiento equilibrado debido a que habían realizado adaptaciones en su estructura y dinámica relacional. Esto generó cambios adaptativos que mejoraron su interacción.

\section{PROCESOS DE COMUNICACIÓN}

Cuando se analizaron los procesos de comunicación que integran la expresión de emociones, diálogo, interacción y sentido del humor, se halló que la mayoría de las familias reportaron que sus emociones podían fluctuar entre el llanto, el enojo, la tristeza... por mencionar algunas de características negativas. Éstas pudieron surgir, en un inicio, por la presencia de la discapacidad y el primer choque emocional. Pero también reconocieron la alegría, el orgullo y sentido a la vida como sensaciones positivas, a través del tiempo.

Ante esta categoría, los hermanos juegan un papel valioso porque ejercitan la expresión de las emociones cuando interactúan entre ellos como iguales; esto no depende del orden de nacimiento. Varios de ellos comentaron en las entrevistas que ha sido complejo y que en ocasiones se han desesperado, se han enojado con sus hermanos con discapacidad o con sus padres, por la manera que habían reaccionado. Pero que paralelamente han encontrado un profundo amor, alegría, significado y satisfacción por los avances de sus hermanos, avances a los cuales ellos han colaborado. 
Según los testimonios de las familias, el diálogo era una forma de comunicarse que no tenían desarrollada al principio de la presencia de la discapacidad, pero que con el tiempo había sido una estrategia que perfeccionaron, poco a poco, debido a que les permitió establecer vías de comunicación entre todos los miembros.

De modo general, las familias entrevistadas manifestaron contar con formas de interacción mediante acciones directas, es decir, que las familias monoparentales que estaban constituidas por madres e hijos o hijas mayores sin discapacidad, establecían formas de interrelación muy intensas, debido a que se convertían en el principal apoyo emocional, físico y social para el manejo de la discapacidad dentro de su sistema, además de ser el sostén para afrontar de modo solidario el quehacer cotidiano.

El vínculo entre ellas y sus hijos o hijas es una fuerza que les ayuda a establecer normas, reglas, acciones, toma de decisiones, labores $\mathrm{o}$ actividades donde participe todo el grupo o algunos miembros de la familia. Así mismo, la interacción con el resto de la familia - la que se denomina «extensa»— es parte del ejercicio cotidiano, debido a que ella se convierte en una red de apoyo que fortalece la identidad y las formas de afrontamiento.

El sentido del humor tiene especial relación en las familias que aportaron sus testimonios, pues se trata de la capacidad de ver lo absurdo en los problemas y encuentra lo cómico en la tragedia (Wolin, 1993, en Puig y Rubio, 2011).

Las madres con hijos pequeños que presentaban una discapacidad, hablaron del juego como el medio con el cual la familia suscita ámbitos para la diversión; otras reportaron que la búsqueda de espacios comunes, los fines de semana, les permitían generar un momento de esparcimiento familiar (como salir a caminar, conversar, andar en bicicleta o visitar a la familia extensa), sin la rutina de la responsabilidad semanal. La familia extensa se convierte en otro lugar de apoyo que no sólo brinda sostén físico, sino también emocional y recreativo. 


\section{NUEVAS ACCIONES EN LA RESILIENCIA FAMILIAR}

Durante las entrevistas se obtuvieron cuatro categorías emergentes, denominadas: singularidad, valores, red de apoyo y espiritualidad. Entre los resultados se encontró en la singularidad que los padres, las madres, los hijos, hermanos, tíos y primos sostenían, en sus afirmaciones, que el reconocimiento a la unicidad de la persona que presenta la discapacidad (hijo, hermano o primo) es proyección de su propia personalidad.

Lo anterior implica que lo identifican como una persona única, no por su condición, sino por su esencia, otorgándole un lugar dentro del grupo familiar, así como una identidad propia. Su trabajo como familias es generar la autonomía en su hijo o hermano, los cuales poseen un valor, reconociendo en ellos su individualidad.

En todas las familias se habló de propiciar ambientes para que la persona con discapacidad desarrolle todo su potencial, así como se distinga y valore que hacen las cosas de distinta manera, por lo tanto, son únicos y no se encuentran en la obligación de realizar las cosas de igual forma que el resto de la sociedad. Para muchos padres ha sido un trabajo de transformación personal, donde el sentido que le otorgan a la adversidad, a su propia narrativa, unida a sus experiencias, les ha permitido lograr esta nueva forma de mirar y distinguir al otro, de reconocer la otredad.

Respeto, servicio, perseverancia, solidaridad, humildad, constancia, responsabilidad, lealtad y fortaleza son algunos valores determinantes en su vida familiar.

La red de apoyo en estas familias es uno de los pilares esenciales, debido a que la mayoría comentó que el grupo de origen es el primer soporte a través del cual se genera la resiliencia, pues están presentes de manera incondicional desde el principio de esta experiencia, además de que su sostén es físico, económico, psicoemocional y colaborativo. Por lo tanto, son el primer clan de respaldo. Los apoyos impulsados por la familia extensa, las amistades y la comunidad aportan soporte emocional e instrumental, de modo que son cruciales y trascendentales para la construcción de la resiliencia familiar (Grau, 2013). 
La vida espiritual es otro de los pilares que, de modo repetido, se observó en el total de familias entrevistadas. En algunas de ellas, sus integrantes expresaron que era la base con la cual podrían dirigir sus vidas y las de sus seres amados.

Finalmente es importante reconocer que la resiliencia familiar es un entramado de muchos fenómenos, donde en el centro puede observarse a la familia como la base sólida que genera una serie de fuerzas intrínsecas que proporcionan dinamismo a todo su entorno.

\section{CONCLUSIONES}

Los testimonios recuperados de los integrantes de las familias entrevistadas, contribuyen con datos significativos al hecho de ser resilientes. En un primer momento, el factor que participa como la base para la construcción de la resiliencia — con el pasar del tiempo y después del enfrentamiento inicial con la discapacidad- es la aceptación, debido a que en todos los entrevistados, las madres y el único padre, reportaron que esta experiencia fue un proceso de asimilación de la condición de su hijo, de manera que poco a poco ésta adquirió un papel determinante ante la nueva circunstancia de vida.

Los miembros de las familias entrevistadas refirieron que la experiencia de la discapacidad ha sido todo un recorrido de emociones y sentimientos contrapuestos, donde las madres aceptan su papel de modo más consciente y voluntario; esto significa que, tanto interna como socialmente, se responsabilizan de su hijo, con miedo, con incertidumbre, con tristeza, pero con una actitud de acogida hacia él. Esta situación parece relacionarse con la aceptación incondicional, la que de modo regular se presenta en la mayor parte de las familias, como un rasgo natural.

El único padre entrevistado confirmó lo que varios autores han descrito en sus investigaciones. De manera personal asumir su papel ante esta situación es complejo, debido a la educación que de modo previo han recibido, así como a la asignación del papel social concedido a los varones que se enfrentan a la discapacidad de un hijo: que no 
pueden o no deben ser los cuidadores principales; sin embargo, el padre entrevistado no se consideraba falto de capacidad.

También desde sus trincheras, los hermanos entrevistados se asumen totalmente resilientes, aun sin saber qué es o lo que significa la palabra, saben que son el apoyo de las madres, que son pieza importante en el desarrollo de su hermano con discapacidad; ellos son los primeros en aceptarlo de modo incondicional, puesto que han recibido el ejemplo de sus madres.

La dinámica del sistema familiar frente a la discapacidad tiene una serie de múltiples factores: desde el reconocimiento de sus miembros, el manejo y la distribución de labores, la colaboración, la adaptación, así como el desarrollo de un conjunto de habilidades y capacidades que nunca habían puesto en práctica. Éstas surgen fortalecidas por la necesidad de salir adelante, es decir, asumen este hecho de la vida con un compromiso total.

Cada familia refirió en las entrevistas que tuvo formas diferentes para alcanzar esta meta, y que en ocasiones ante las transformaciones que se presentan, generaban formas creativas de afrontamiento. Nunca desisten, tienen altibajos propios de la naturaleza humana, pero se encuentran en la lucha, debido a que así lo refieren: como una «lucha constante».

La figura central —insistimos — sigue siendo la madre; juega un papel de gran impacto al ser ella autodeterminada. Otorgan una mirada diferente a la discapacidad de sus hijos, fortalecida por los discursos internos que se repiten de modo constante y que traducen en acciones. La gran mayoría no había experimentado una crisis de tal envergadura, por lo cual no sabían hasta dónde eran capaces de lograr sus metas, por lo tanto, mostraron tener conciencia de la confianza en sí mismas.

La experiencia de la discapacidad en estas familias permite inferir que la resiliencia es comprendida como un proceso en evolución, el cual surge de modo individual, pero se erige conforme se suscita la relación con otros. Esta interacción establece una directriz a través del enfrentamiento individual: el primer paso es la aceptación, 
la ambivalencia (experiencias/expectativas), un sentido otorgado a la adversidad, las narrativas internas, el reconocimiento a la singularidad del miembro con discapacidad y la vida espiritual.

Las acciones previas - particularmente el sentido frente a la adversidad, las narrativas, la singularidad y la vida espiritual - se reiteran y fortalecen dentro de la familia, todas generan resiliencia familiar, la cual se robustece con la comunicación, el diálogo, el apoyo y la cohesión familiar, además del sentido del humor, la vivencia de valores y la estructura que posean (funcionalidad/adaptabilidad). Para que todo vinculado conduzca a la concepción de una resiliencia social, donde los recursos son el apoyo social de la familia, amigos, profesionales, escuela y comunidad, es decir acciones de carácter colectivo y compartido.

Por lo tanto, se afirma que la resiliencia es un proceso dinámico que en las familias puede surgir mediante diversos aspectos de la vida en grupo. Queda en evidencia que la construcción de la resiliencia es un proceso que se va presentando paulatinamente.

De modo efectivo, ante esta experiencia, las familias desarrollan un conjunto de habilidades y capacidades nunca antes puestas en práctica. Éstas surgen fortalecidas por la necesidad de salir adelante, es decir, lo asumen con responsabilidad total, reconociendo la identidad del hijo con discapacidad y su inclusión en la sociedad. De aquí la importancia del enfoque centrado en la familia, pues las empodera.

En la figura 2 se describen las conclusiones generadas durante este proceso de construcción de la resiliencia, en familias que viven la discapacidad en un hijo. 


\section{Figura 2}

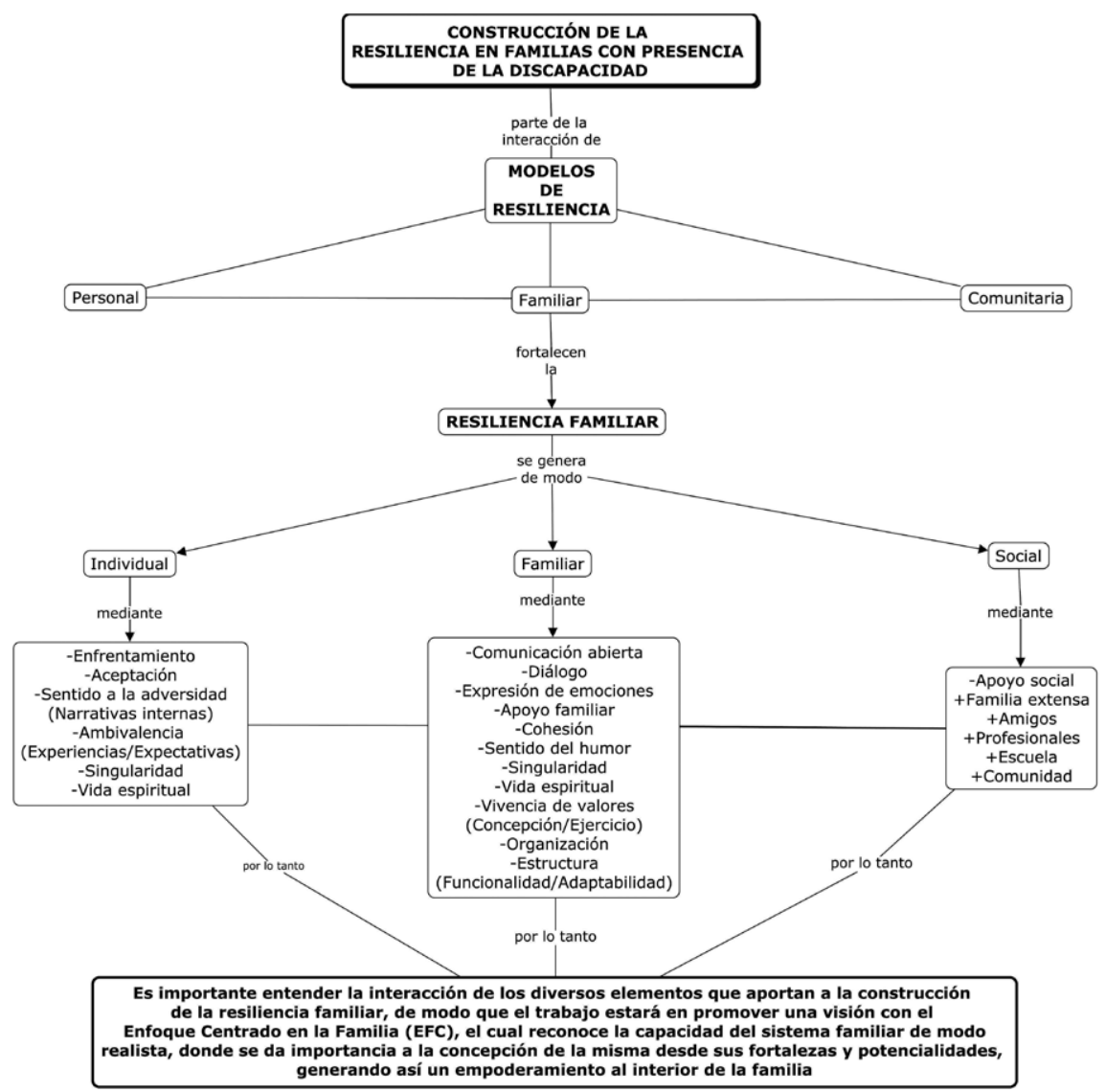

Fuente: Elaboración propia.

Con la información obtenida, surge la necesidad de continuar con la investigación de esta temática - particularmente con el conocimiento en el impacto, la influencia y el sentir de los hermanos no diagnosticados- para recuperar la experiencia de los padres varones implicados en la educación de sus hijos, así como en la manera de generar apoyo para las madres solas y sin sostén familiar, a partir de sus propios tiempos, preparación profesional y condiciones particulares. Esto permitirá aportar conocimiento para apoyar a las familias, así como a los profesionales del área. 


\section{REFERENCIAS}

Anthony, E.J. (1987). Risk, vulnerability, and resilience: An overview. The invulnerable child, 3-48. Recuperado de https:// s3.amazonaws.com/academia.edu.documents/29815627/Anthony Invulnerable.pdf? AWSAccessKeyId=AKIAIWOWYYGZ2Y53UL3A\&Expires $=1504445132 \&$ Signature $=\mathrm{HO} \%$ 2BQHzo25jraZ25R\%2BX\%2BK5YO7JNY\%3D\&response-content-disposition $=$ inline $\% 3 \mathrm{~B} \%$ 20filename\%3DRisk Vulnerability and Resilence An Over.pdf

Araya, M. y Castro, G. (2012). La familia como promotora de la independencia en niños y niñas con discapacidad motora. Revista Electrónica Educare, 16 (1), 99-111. Recuperado de http:/ / www. redalyc.org/html/1941/194124281009/

Aznar, A. y González, D, (2008). ¿Son o se hacen? El campo de la discapacidad intelectual estudiado a través de recorridos múltiples. Buenos Aires: Noveduc.

Bayat, M. (2010). Evidence of resilience in families if children with autism. Journal of Intellectual Disability Research, 51(9), 702-714. Recuperado de https://s3.amazonaws.com/academia.edu.documents / 8061741/ Evidence\% 20of\%20resilience. pdf?AWSAccessKeyId=AKIAIWOWYYGZ2Y53UL3A\&Expires $=1504226333 \&$ Signature $=3$ y RGC0A1p0KJbYAUnlUWSdaajo\%3D\&response-content-disposition=inline $\% 3 \mathrm{~B} \% 20$ filename\%3DEvidence of resilience in families_of_ch.pdf

Blacher, J. y Baker, B. (2007). Positive impacto f intelectual disability on families. American Journal on Mental Retardation, 112, 330-348.

Calero, J. (2012). La «primera noticia» en familias que reciben un hijo con discapacidad o problemas en el desarrollo. Algunas estrategias de afrontamiento. Revista EDETANIA, 41(45-56) ISSN: 0214-8560. Recuperado de https://dialnet.unirioja.es/servlet/ articulo? codigo $=4089659$ 
De Andrade Seidl, M.L., y Da Cruz Benetti, S.P. (2011). Resiliencia familiar: nuevas perspectivas en la promoción y prevención en salud. Diversitas: Perspectivas en Psicología, 7(1). Recuperado de http:/ / www.redalyc.org/html/679/67922583004/

Delage, M. (2010). La resiliencia familiar. El nicho familiar y la superación de las heridas. España: Gedisa.

Dempsey, I., y Keen, D. (2008). Una revisión de los procesos y resultados en los servicios centrados en la familia para niños con una discapacidad. Temas en la educación especial de la primera infancia, 28 (1), 42-52. Recuperado de https:// s3.amazonaws.com/academia.edu.documents/30861046/42. pdf?AWSAccessKeyId=AKIAIWOWYYGZ2Y53UL3A\&Expires $=1504636211 \&$ Signature $=$ AVARtdp6FEUw9F6bHTLNv0sqFB8\%3D\&response-content-disposition $=$ inline $\% 3 \mathrm{~B} \% 20$ filename\%3DA_review of processes and outcomes in fa.pdf

Dunst, C.J., Trivette, C.M., y Deal, AG. (1989). Permitir y empoderar a las familias: Principios y directrices para la práctica. EU. Libros de Brookline. https:// doi.org/10.1002/1520-6807(198910)26:4<4 24::AID-PITS2310260416>3.0.CO;2-6

Dunst, C.J., Trivette, C.M., y Hamby, D.W. (2007). Meta-análisis de investigaciones de prácticas de ayuda centradas en la familia. Retrasos mentales y discapacidades del desarrollo Research Reviews, 13 (4), 370-378. http://dx.doi.org/10.1002/mrdd.20176

Eguiluz, L. (2003). Dinámica de la familia. Un enfoque psicológico sistémico. México: Pax México.

Garmezy, N. (1991). Resiliencia y vulnerabilidad a los resultados adversos del desarrollo asociados con la pobreza. American behavioral scientist, 34 (4), 416-430.

Garzón, K. (2010). Discapacidad y procesos identitarios. Revista Ciencias de la Salud, 5(2). Recuperado de http: / / revistas.urosario. edu.co/index.php/revsalud/article/view/731 
Giné, C., Gràcia, M., Vilaseca, R., y Balcells, A. (2009). Trabajar con las familias en atención temprana. Revista interuniversitaria de formación del profesorado, 23(2). Recuperado de http://www. redalyc.org $/ \mathrm{html} / 274 / 27419063008 /$

Grau, C. (2013). Fomentar la resiliencia en familias con enfermedades crónicas pediátricas. Revista española de discapacidad, 2013, vol. I, núm. I, p. 195-212. Recuperado de http:/ / www.cedd.net/ redis/index.php/redis/article/viewFile/42/34

Grotberg, E. (2006). La resiliencia en el mundo de hoy. Cómo superar las adversidades. Barcelona: Gedisa.

Grotberg, E. H. (1995). A guide to promoting resilience in children: Strengthening the human spirit. The Hague, Netherlands: Bernard van Leer Foundation.

Kotliarenco, M.A., Cáceres, I., y Fontecilla, M. (1997). Estado de arte en resiliencia. Washington D.C.: Organización Panamericana de la salud. Recuperado de http://200.32.31.245/transferencia-universitaria/aprendizaje-y-servicio/ESTADO $\% 20 \mathrm{DE} \% 20$ ARTE \%20EN\%20RESILIENCIA \%20-\%20Organizacion \%20Panamericana $\% 20 \mathrm{de} \% 201 \mathrm{a} \% 20$ Salud $\% 20-\% 20$ Organizacion $\% 20$ Mundial $\% 20$ de $\% 201 \mathrm{a} \% 20$ Salud.pdf

León, S. (2015). La perspectiva familiar sistémica en la medicina general. MéD. UIS, 28(1), 159-162. Recuperado de file:/ / C:/ Users/v ferez/Downloads/Dialnet-LaPerspectivaFamiliarSistemicaEnLaMedicinaGeneral-5168239\%20(4).pdf

López, Silvia; y Escudero, V. (2003). Familia, evaluación e intervención. Alcalá, España: CCS.

Luthar, SS, y Zigler, E. (1991). Vulnerabilidad y competencia: una revisión de la investigación sobre la resiliencia en la infancia. Revista estadounidense de Orthopsychiatry, 61 (1), 6.

Macías, R. (2012). Entendiendo y tratando el corazón de la familia. Un modelo dinámico, sistémico, integrativo. México: El Saber Instituto. 
McCubbin, M., Balling, K., Possin, P., Frierdich, S. y Bryne, B. (2002), Resiliencia familiar de cáncer infantil. Recuperado de https: / / onlinelibrary.wiley.com/doi/ full/10.1111/j.1741-3729.200 2.00103.x\#fn1 Family Relations, 51: 103-111. doi: 10.1111 / j.17413729.2002.00103.x

Mercado-García, E., Aizpurúa-González, E. y García-Vicente, L. (2012). Características, percepciones y necesidades sociales de los niños y niñas con discapacidad y sus familias. Portularia, XII(2) 69-80. Recuperado de http:/ / www.redalyc.org/articulo. oa?id=161024690006

Mora, C. (2012). Familias de pacientes con parálisis cerebral severa: sus indicadores de calidad de vida. Investigación y Ciencia, 20(55). Recuperado de http://www.redalyc.org/ html/674/67424409004/

Minuchin, S. (2008). Familias y terapia familiar. Barcelona: Gedisa.

Ortega, P., Salguero, A., y Garrido, A. (2007). Discapacidad: paternidad y cambios familiares. Avances en Psicología Latinoamericana, 25(1). Recuperado de http://www.redalyc.org/ $\underline{\mathrm{html} / 799 / 79902511 /}$

Ortega, P., Torres, L., Reyes, A., y Garrido, A. (2010). Paternidad: niños con discapacidad. Revista Intercontinental de Psicología y Educación, 12(1) 135-155. Recuperado de http://www.redalyc. org/articulo.oa?id $=80212393008$

Osborn, A. (1993). What is the value of the concept of resiliencie for policy and intervention. Londres: Internacional Catholic Chil Burea.

Palomar, J., y Gaxiola, J. (2012). Estudios de resiliencia en América Latina volumen 1. México: Pearson.

Palomar Lever, J., y Gómez Valdez, N.E. (2010). Desarrollo de una escala de medición de la resiliencia con mexicanos (RE- 
SI-M). Interdisciplinaria, 27(1), 7-22. Recuperado de http:/ / www. redalyc.org/articulo.oa?id=18014748002

Patterson, JM (1991). Resistencia de la familia al desafío de la discapacidad de un niño. Anales pediátricos, 20 (9), 491-499. Recuperado de https://www.healio.com/pediatrics/journals/ pedann/1991-9-20-9/\%7B00cf5d6e-0688-49c0-baf9-708b95c4d$\underline{\text { 83d\%7D/family-resilience-to-the-challenge-of-a-childs-disabili- }}$ ty DOI: 10.3928 / 0090-4481-19910901-08

Peralta, F., y Arellano, A. (2010). El papel de la familia en la promoción de la autodeterminación de sus hijos/as con Síndrome de Down: el Enfoque Centrado en la Familia (ECF). En Ministerio de Sanidad, Política Social e Igualdad (Ed.) Actas del II Congreso Iberoamericano sobre el Síndrome de Down: «La Fuerza de la Visión Compartida» (pp. 835-844) Granada, España. Recuperado de http:// www.centrodocumentaciondown.com/uploads/documentos/ e4dd9d6b4249e550046bd57e9aa942f027efa50d.pdf.

Peralta, F. (2012). Abordaje familiar y profesional de la discapacidad cognitiva: la autodeterminación personal y la planificación centrada en la persona. Departamento de Educación. Universidad de Navarra España. Recuperado de http:// www.descubreme.cl/wp-content/uploads/2012/05/Abordaje-familiar-y-profesional-de-la-discapacidad-cognitiva-la-autodeterminaci\% C3\%B3n-personal-_Feli-Peralta_.pdf

Puig, G. y Rubio, J. (2011). Manual de resiliencia aplicada. Barcelona: Gedisa.

Roque, M. D. P., y Acle, G. (2013). Resiliencia materna, funcionamiento familiar y discapacidad intelectual de los hijos en un contexto marginado. Univ. Psychol. Bogotá, Colombia, 12(3), 811-820. Recuperado de http:/ / www.redalyc.org/html/647/64730275013/

Rutter, M. (1993). Resiliencia: Algunas consideraciones conceptuales. Revista de salud del adolescente. Volumen 14, Número 8, 626-631. 
Rutter, M (1985). Resilience in the face of adversity: Protective factors and resistance to psychiatric disorder. British Journal of Psychiatric, 147, 598-611. Recuperado de http:/ / bjp.rcpsych.org/ content $/ 147 / 6 / 598$

Suriá, R. (2013) Discapacidad y empoderamiento: análisis de esta potencialidad en función de la tipología y etapa en la que se adquiere la discapacidad. Anuario de psicología, 43(3). Recuperado de http: / / www.redalyc.org/html/970/97030658002/

Trute, B., Hiebert-Murphy, D., y Levine, K. (2007). Parental appraisal of the family impact of childhood developmental disability: Times of sadness and times of joy. Journal of Intellectual and Developmental Disability, 32(1), 1-9. Recuperado de https:/ / www.researchgate.net/profile/Diane_Hiebert-Murphy / publication/6442645 Parental_appraisal_of the family impact_of childhood developmental disability Times of sadness and times of joy/links/00b7d51d2cfc10bcff000000/ Parental-appraisal-of-the-family-impact-of-childhood-developmental-disability-Times-of-sadness-and-times-of-joy.pdf

Vargas, M. (2013). Resiliencia familiar y desarrollo del descendiente con síndrome de Down. Revista UABC, (72). Recuperado de http://www.sindromedown.net/wp-content/ uploads/2014/09/146L_14.pdf

Vanistendeal, S. (2014). Resiliencia: el reto del cambio de mirada. En J. Madariaga. Nuevas miradas sobre la resiliencia. Ampliando ámbitos y prácticas (pp. 53-67). Barcelona: Editorial Gedisa.

Villalba, C. (2003). El concepto de resiliencia individual y familiar. Aplicaciones en la intervención social. Psychosocial Intervention, 12(3) 283-299. Recuperado de http://www.redalyc.org/articulo.oa?id=179818049003

Walsh. F. (2004). Resiliencia familiar: estrategias para su fortalecimiento. Madrid: Amorrortu. 
Werner, E., y Smith, R. (1992). Overcoming the odds: High risk children from birth to adulthood. Cornell University Press.

Wolin, S. Wolin (1993). El yo resistente: cómo los sobrevivientes de las familias con problemas se elevan por encima de la adversidad. Nueva York: Villard. 